\title{
Debonding Time and Dental Pulp Temperature With the Er, Cr: YSGG Laser for Debonding Feldespathic and Lithium Disilicate Veneers
}

\author{
Marzieh Alikhasi ${ }^{1}$, Abbas Monzavi ${ }^{2}$, Hooman Ebrahimi ${ }^{3}$, Maryam Pirmoradian $^{4}$, Ahmadreza Shamshiri $^{5}$, \\ Rezvaneh Ghazanfari' ${ }^{6}$
}

${ }^{1}$ Dental Laser Research Center, Dental Research Center, Dentistry Research Institute, Department of Prosthodontics, School of Dentistry, Tehran University of Medical Sciences, Tehran, Iran

${ }^{2}$ Dental Laser Research Center, Dentistry Research Institute, Department of Prosthodontics, School of Dentistry, Tehran University of Medical Sciences, Tehran, Iran

${ }^{3}$ Department of Oral Medicine, Dental Branch, Islamic Azad University, Tehran, Iran

${ }^{4} \mathrm{PhD}$ Candidate, Department of Dental Biomaterials, School of Dentistry, Research Center for Science and Technology in Medicine, Tehran University of Medical Sciences, Tehran, Iran

${ }^{5}$ Department of Epidemiology and Biostatistics, School of Public Health and Institute of Public Health Research, Tehran University of Medical Sciences, Tehran, Iran

${ }^{6}$ Department of Prosthodontics, School of Dentistry, International Campus, Tehran University of Medical Sciences, Tehran, Iran

\section{*Correspondence to Rezvaneh Ghazanfari, DDS, MS; Assistant Professor, Department of Prosthodontics, School of Dentistry, International Campus, Tehran University of Medical Sciences, Tehran, Iran. \\ Tel: 009855851151 \\ Email: rezvane_aurora66@yahoo. com}

Published online July 6, 2019

\begin{abstract}
Introduction: The removal of ceramic veneers is a time-consuming procedure in a dental office. Little research has been done in alternative removal techniques for ceramic veneers. The objective of this study was to evaluate the removal of feldspathic and lithium disilicate reinforced glass ceramic veneers by Er, Cr: YSGG and to measure debonding time and pulpal temperature increase during veneer removal.

Methods: Fifty-seven bovine incisor teeth were prepared and divided into 3 groups. Ceramic specimens with a thickness of $0.7 \mathrm{~mm}$, a width of $4 \mathrm{~mm}$ and a length of $8 \mathrm{~mm}$ were fabricated from feldspathic ceramic, lithium disilicate reinforced glass ceramic HT (high translucency) and lithium disilicate reinforced glass ceramic $\mathrm{MO}$ (medium opacity) (19 for each group). Specimens were cemented on the labial surface of incisors using resin cement. The Er, Cr: YSGG laser was applied to each specimen at $2.5 \mathrm{~W}$ and $25 \mathrm{~Hz}$. Debonding time was measured for each specimen, and the intrapulpal temperature was detected in 3 specimens for each group. Data were analyzed via one-way analysis of variance (ANOVA) at significance level of $0.05(\mathbb{\nabla}=0.05)$. Results: Mean debonding time was 103.68 (26.76), 106.58 (47.22) and 103.84 (32.90) seconds for feldspathic, lithium disilicate $\mathrm{MO}$, and lithium disilicate HT respectively. There was no significant statistical difference among the groups ( $\mathrm{P}$ value $=0.96$ ). The intrapulpal temperature increase was less than $1^{\circ} \mathrm{C}$ in all groups.

Conclusion: Er, Cr: YSGG can successfully be used to efficiently debond feldspathic and lithium disilicate reinforced glass ceramic veneers. There was no significant difference for debonding time among these ceramic materials. During ceramic laminate veneer removal by laser irradiation, no irritating temperature rise was detected.

Keywords: Porcelain Laminate Veneer; Er, Cr: YSGG Laser; Debonding.
\end{abstract}

\section{Introduction}

Laminate veneers have many indications to improve appearance, such as discoloration, hypocalcification, peg lateral teeth, fluorosis, ${ }^{1}$ fractured teeth, and diastema. ${ }^{2}$ These restorations are firmly attached to labial surfaces of anterior teeth by different kinds of resin-based cement. Under different clinical circumstances, light or dual cure resin cement may be selected. ${ }^{3}$

The longevity of veneers is limited by reason of many factors such as discoloration and microleakage of resin cement and marginal fracture of restorations. ${ }^{4}$ Under these conditions, veneers have to be removed from tooth surfaces. The conventional removal procedure is done by grinding these restorations with rotary instruments, 
although it is time-consuming and inconvenient for the patient and damaging for the dental structure..$^{5}$ A great number of studies have used different types of lasers $(\mathrm{Nd}$ : YAG, Er: YAG, $\mathrm{CO}_{2}$, Tm: Yap, diode and yttrium fiber lasers) for debonding ceramic brackets in an efficient way by reducing shear bond strength of ceramic bracket and debonding time..$^{5-8}$ Under the light of these studies, investigations have been done to study the use of Er, YAG for the removal of ceramic veneers and even ceramic crowns.

Erbium lasers including erbium, chromium-doped yttrium, scandium, gallium and garnet laser (Er: CrYSGG) and erbium-doped yttrium, aluminium and garnet laser (Er:YAG) have an emission wavelength of $2780 \mathrm{~nm}$ and $2940 \mathrm{~nm}$ respectively, which correlates with the major absorption peak of water, hydrated tissues, residual monomers and bonding cement containing water. ${ }^{9-11}$ Recent investigations have shown that the Er:CrYSGG has three times lower absorption rate than the Er:YAG, and this can be attributed to the difference in the water content of dentin and enamel. Because of its higher absorption rate, the Er:YAG laser has a lower penetration depth and therefore requires less energy and less time to ablate the tissue. ${ }^{12,13}$

The laser-aided ceramic veneers removal has many advantages and several clinical factors may affect it, including chemical composition and type of ceramic, thickness of restoration, resin cement type and shade, ceramic shade and opacity, and laser parameters such as power, pulse duration, frequency and irradiation time. ${ }^{11,14-18}$

The optical characteristic of the restoration to be debonded by laser irradiation is a determining factor in this procedure and optical features in different types of ceramics are greatly determined by the crystalline phase of ceramic. Ceramic veneers (feldespathic ceramic and lithium disilicate glass ceramic) consist of different crystalline phases. ${ }^{19}$ In addition, it has been shown that depending on ceramic thickness, feldespathic ceramic and lithium disilicate glass ceramic have different rates of Er,YAG laser energy transmission (60\% and $40 \%$ respectively). ${ }^{14,20}$ Therefore, the objective of this laboratory study was to compare the effect of the Er,Cr:YSGG laser on debonding time of three ceramic veneers and dental pulp temperature.

\section{Methods}

Fifty-seven extracted, noncarious, permanent bovine mandibular incisor teeth were selected and divided into 3 groups. Roots were cut off and cylindrical diamond bur was used to make a flat surface on the labial aspect of teeth. Then, the surfaces were polished, rinsed and dried with air. Enamel surfaces were etched with 37\% phosphoric acid for 30 seconds, then washed and dried.

The ceramic veneer materials used in this study were emax Press HT and MO (Emax) (lithium disilicate glass ceramic high translucency (HT) and medium opacity (MO); Ivoclar, Vivadent, Switzerland) and Ceramco I.C (feldspathic ceramic), and shade A2 was used for all groups. Nineteen Ceramic specimens of each material with flat surfaces $(4 \mathrm{~mm} \times 8 \mathrm{~mm}$, an average thickness of $0.7 \pm 0.05 \mathrm{~mm}$ ) were produced in a dental laboratory according to the manufacturer's instruction.

Specimens were cemented to the labial surfaces of incisors using Variolink N LC (Ivoclar, Vivadent) by light finger pressure for all veneers and light cured for 20 seconds. Before debonding, the samples were stored in distilled water at $37^{\circ} \mathrm{C}$ for 48 hours.

For debonding, the Er,Cr:YSGG $(2870 \mathrm{~nm})$ laser with pulse duration of $60 \mu \mathrm{s}$ was applied to each specimen at $2.5 \mathrm{~W}$ and $25 \mathrm{~Hz}$. In this study, the application tip (MZ6, $600 \mu \mathrm{m}$, quartz tip [biolased technology]) was positioned perpendicularly at a $2-\mathrm{mm}$ distance from the ceramic veneers and scanning method was performed with horizontal movements parallel to the surface. ${ }^{7}$

Debonding time was measured for each specimen and the intrapulpal temperature was continuously detected with K-type thermocouple in 3 specimens of each group. The tip of the thermocouple was positioned in the pulp chamber directly under the veneer (Figure 1). One-way analysis of variance (ANOVA) was used for comparison of the groups. A statistically significant difference was considered to be $P<0.05$.

\section{Results}

Mean debonding time was 103.68 (26.76), 106.58 (47.22) and 103.84 (32.90) seconds for feldspathic, lithium disilicate $\mathrm{MO}$, and lithium disilicate HT respectively (Table 1). One-way ANOVA revealed no significant difference among debonding time of the three groups of the study $(P>0.05)$.

In the Emax MO and Emax HT group, no fracture happened during debonding and the veneers slid off in one piece. In the feldspathic group, many cracks happened in all veneers which could be detected from the

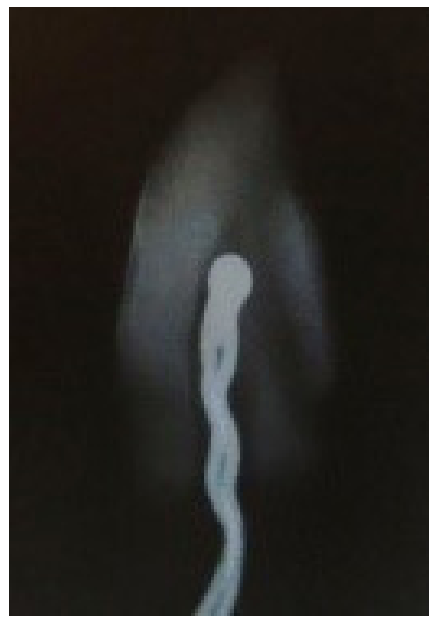

Figure 1. Position of the Tip of Thermocouple in the Pulp Chamber. 
Table 1. Mean, Standard Deviation, Minimum and Maximum Debonding Time of the Study Groups in Second

\begin{tabular}{lcccc}
\hline \multirow{2}{*}{ Ceramic } & \multicolumn{4}{c}{ Time } \\
\cline { 2 - 5 } & Mean & Std. Deviation & Minimum & Maximum \\
\hline Feldspathic & 103.68 & 26.76 & 60.00 & 170.00 \\
Emax MO & 106.58 & 47.22 & 10.00 & 213.00 \\
Emax HT & 103.84 & 32.90 & 47.00 & 178.00 \\
\hline
\end{tabular}

first seconds of irradiation to the end. In this group, all veneers were removed in more than 4 pieces.

In all groups, only very small remnants of cement carbonization were detected on ceramic veneers. The Intrapulpal temperature increase was less than $1^{\circ} \mathrm{C}$ in all groups.

\section{Discussion}

The use of laser energy for ceramic veneer removal requires a transition of laser energy, energy absorption by resin cement, and degradation of cement by one of the three presumed mechanisms of ceramic bracket removal, thermal softening, thermal ablation, and photoablation. ${ }^{21}$ In the thermal softening process, the bonding agent is heated until it softens and the bracket slides off the tooth surface. Thermal ablation occurs when the temperature increases rapidly in an adhesive resin vaporization range. As a result, the bracket blows off the tooth surface before thermal softening occurs. In photoablation, the energy level of the bonds between the bonding-resin atoms rapidly increases above their dissociation energy levels, resulting in the decomposition of the material. In comparison, thermal softening occurs at low power of densities - thermal ablation and photoablation occur at high power densities. ${ }^{22}$ Oztoprak et al stated that laminate veneer removal by the scanning method did not cause explosive blow off, and this means that thermal ablation and photoablation are not causative mechanisms. They also assumed that this process did not occur only by reason of thermal softening, and physical disruption in resin cement may be an underlying mechanism of degradation as well..$^{5}$ In addition, Morford et al reported that laser ablation rather than thermal softening resulted in debonding. ${ }^{23}$ In line with recent studies, we investigated that not only thermal softening but also physical disruption and cement ablation can be considered as underlying mechanisms of porcelain laminate veneer removal.

The mode of failure in the debonding process is an indicative factor of enamel damage. Failures occur in enamel/cement interface, increasing the risk of enamel damage. Oztoprak et al reported that in their study the majority of failures occurred in cement and enamel fracture was not investigated. ${ }^{5}$ Using microscopic images, Morford et al stated that the majority of failures occurred in the veneer/cement interface and veneer surfaces were clean and free of cement. They also found no sign of ablation or even ablation crater in enamel, the reason of which was the energy density which was 20 times lower than energy density needed for enamel ablation. ${ }^{23}$ In this study, the main bulk of resin cement remained on the tooth surface. This investigation showed that laseraided porcelain laminate veneer removal might be done without enamel damage. In line with recent studies, ${ }^{20,24}$ we also found that laser irradiation had no effect on ceramic surfaces.

The results of this study showed that there was no difference in debonding time of feldspathic ceramic and lithium disilicate reinforced glass ceramic of two types of MO and HT. In contrast, Sari et al showed that different kinds of ceramics differed in the energy transition rate. Sixty-eight percent of laser energy passes through feldspathic ceramic of $0.5 \mathrm{~mm}$ and $88 \%$ of energy passes through the same thickness of lithium disilicate reinforced glass ceramic. ${ }^{20}$ The reasons for these conflicting results include different types of lasers, different laser power and frequency and different study conditions.

One of the major concerns of laser-aided debonding is pulpal temperature increase during laser irradiation. Currently, in dental sciences, pulpal temperature increase more than $5.5^{\circ} \mathrm{C}$ is considered as critical and irritating for dental pulp, and this temperature was first stated by Zach and Cohen in 1965. In this study, temperature increase was less than $1^{\circ} \mathrm{C}$ in all specimens. In this study, the scanning method ${ }^{7}$ was used, which may be one of the most important factors of low temperature increase in this study. In addition, laser type and parameters, cooling air and water spray led to less thermal effects.

\section{Conclusion}

Investigating the effect of the Er,Cr:YSGG laser on debonding time of ceramic veneers and dental pulp temperature, the present study showed that the application of Er,Cr:YSGG was an efficient, fast and safe method for the removal of both feldspathic and lithium reinforced glass ceramic porcelain laminate veneers. There was no significant difference in debonding time of these ceramic materials. During ceramic laminate veneer removal by laser irradiation, temperature rises exceeding $5.5^{\circ} \mathrm{C}$ were not detected.

\section{Ethical Considerations}

Not applicable.

\section{Conflict of Interests}

The authors declare no conflict of interest.

\section{References}

1. Azzaldeen A, Muhamad AH. Restoration of esthetics using ceramics laminate veneer; clinical review: a case report. $J$ Adv Med Dent Sci Res. 2015;3(1):180-185.

2. Magne P, Hanna J, Magne M. The case for moderate "guided prep" indirect porcelain veneers in the anterior 
dentition. The pendulum of porcelain veneer preparations: from almost no-prep to over-prep to no-prep. Eur J Esthet Dent. 2013;8(3):376-388.

3. Runnacles P, Correr GM, Baratto Filho F, Gonzaga CC, Furuse AY. Degree of conversion of a resin cement lightcured through ceramic veneers of different thicknesses and types. Braz Dent J. 2014;25(1):38-42.

4. Kursoglu P, Gursoy H. Removal of fractured laminate veneers with Er:YAG laser: report of two cases. Photomed Laser Surg. 2013;31(1):41-43. doi:10.1089/pho.2012.3410

5. Nalbantgil D, Oztoprak MO, Tozlu M, Arun T. Effects of different application durations of ER:YAG laser on intrapulpal temperature change during debonding. Lasers Med Sci. 2011;26(6):735-740. doi:10.1007/s10103-0100796-7

6. Mundethu AR, Gutknecht N, Franzen R. Rapid debonding of polycrystalline ceramic orthodontic brackets with an Er:YAG laser: an in vitro study. Lasers Med Sci. 2014;29(5):1551-1556. doi:10.1007/s10103-013-1274-9

7. Oztoprak MO, Nalbantgil D, Erdem AS, Tozlu M, Arun T. Debonding of ceramic brackets by a new scanning laser method. Am J Orthod Dentofacial Orthop. 2010;138(2):195200. doi:10.1016/j.ajodo.2009.06.024

8. Nalbantgil D, Tozlu M, Oztoprak MO. Pulpal thermal changes following Er-YAG laser debonding of ceramic brackets. Sci World J. 2014;2014:912429. doi:10.1155/2014/912429

9. Iseri U, Oztoprak MO, Ozkurt Z, Kazazoglu E, Arun T. Effect of Er:YAG laser on debonding strength of laminate veneers. Eur J Dent. 2014;8(1):58-62. doi:10.4103/13057456.126243

10. Oztoprak MO, Tozlu M, Iseri U, Ulkur F, Arun T. Effects of different application durations of scanning laser method on debonding strength of laminate veneers. Lasers Med Sci. 2012;27(4):713-716. doi:10.1007/s10103-011-0959-1

11. Rechmann P, Buu NC, Rechmann BM, Finzen FC. Laser all-ceramic crown removal and pulpal temperature-a laboratory proof-of-principle study. Lasers Med Sci. 2015;30(8):2087-2093. doi:10.1007/s10103-015-1738-1

12. Hibst R, Keller U. Mechanism of Er:YAG laser-induced ablation of dental hard substances. Paper presented at: Lasers in Orthopedic, Dental, and Veterinary Medicine II; 1993. doi:10.1117/12.148318

13. Mirhashemi A, Chiniforush N, Jadidi H, Sharifi N. Comparative study of the effect of Er:YAG and Er:Cr;YSGG lasers on porcelain: etching for the bonding of orthodontic brackets. Lasers Med Sci. 2018;33(9):19972005. doi:10.1007/s10103-018-2573-y
14. Rechmann P, Buu NC, Rechmann BM, Le CQ, Finzen FC, Featherstone JD. Laser all-ceramic crown removal-a laboratory proof-of-principle study-phase 1 material characteristics. Lasers Surg Med. 2014;46(8):628-635. doi:10.1002/lsm.22279

15. Ekworapoj P, Sidhu SK, McCabe JF. Effect of different power parameters of Er,Cr:YSGG laser on human dentine. Lasers Med Sci. 2007;22(3):175-182. doi:10.1007/s10103006-0426-6

16. Corona SA, de Souza AE, Chinelatti MA, Borsatto MC, Pecora JD, Palma-Dibb RG. Effect of energy and pulse repetition rate of Er: YAG laser on dentin ablation ability and morphological analysis of the laser-irradiated substrate. Photomed Laser Surg. 2007;25(1):26-33. doi:10.1089/pho.2006.1075

17. Corona SA, Souza-Gabriel AE, Chinelatti MA, Pecora JD, Borsatto MC, Palma-Dibb RG. Influence of energy and pulse repetition rate of Er:YAG laser on enamel ablation ability and morphological analysis of the laser-irradiated surface. J Biomed Mater Res A. 2008;84(3):569-575. doi:10.1002/jbm.a.31335

18. Raucci-Neto W, Chinelatti MA, Palma-Dibb RG. Ablation rate and morphology of superficial and deep dentin irradiated with different Er:YAG laser energy levels. Photomed Laser Surg. 2008;26(6):523-529. doi:10.1089/ pho.2007.2201

19. Van Noort R, Barbour ME. Introduction to dental materials. 4th ed. Elsevier Health Sciences; 2013.

20. Sari T, Tuncel I, Usumez A, Gutknecht N. Transmission of Er:YAG laser through different dental ceramics. Photomed Laser Surg. 2014;32(1):37-41. doi:10.1089/pho.2013.3611

21. Han X, Liu X, Bai D, Meng Y, Huang L. Nd:YAG Laseraided ceramic brackets debonding: Effects on shear bond strength and enamel surface. Appl Surf Sci. 2008;255(2):613-615. doi:10.1016/j.apsusc.2008.06.082

22. Tocchio RM, Williams PT, Mayer FJ, Standing KG. Laser debonding of ceramic orthodontic brackets. Am J Orthod Dentofacial Orthop. 1993;103(2):155-162. doi:10.1016/ s0889-5406(05)81765-2

23. Morford CK, Buu NC, Rechmann BM, Finzen FC, Sharma AB, Rechmann P. Er:YAG laser debonding of porcelain veneers. Lasers Surg Med. 2011;43(10):965-974. doi:10.1002/lsm.21144

24. Tak O, Sari T, Arslan Malkoc M, Altintas S, Usumez A, Gutknecht N. The effect of transmitted Er:YAG laser energy through a dental ceramic on different types of resin cements. Lasers Surg Med. 2015;47(7):602-607. doi:10.1002/lsm.22394 